\title{
Plagiarism in South African management journals
}

AUTHORS:

Adele Thomas ${ }^{1}$

Gideon P. de Bruin ${ }^{1}$

\section{AFFILIATION:}

'Department of Industrial

Psychology and People

Management, University of

Johannesburg, Johannesburg,

South Africa

\section{CORRESPONDENCE TO:}

Adele Thomas

\section{EMAIL:}

adelet@uj.ac.za

\section{POSTAL ADDRESS:}

Department of Industrial

Psychology and People

Management, University

of Johannesburg, PO Box

524, Auckland Park 2006,

South Africa

\section{DATES:}

Received: 14 Jan. 2014

Revised: 21 July 2014

Accepted: 23 July 2014

\section{KEYWORDS:}

academic values; ethics; government subsidy; reputation; universities

\section{HOW TO CITE:}

Thomas A, De Bruin GP. Plagiarism in South African management journals. $S$ Afr J Sci. 2015;111(1/2), Art. \#2014-0017, 3 pages. http://dx.doi.org/10.17159/ sajs.2015/20140017

(C) 2015. The Author(s). Published under a Creative Commons Attribution Licence.
Plagiarism by academics has been relatively unexplored thus far. However, there has been a growing awareness of this problem in recent years. We submitted 371 published academic articles appearing in 19 South African management journals in 2011 through the plagiarism detection software program Turnitin ${ }^{\mathrm{TM}}$. High and excessive levels of plagiarism were detected. The cost to government of subsidising unoriginal work in these journals was calculated to approximate ZAR7 million for the period under review. As academics are expected to role model ethical behaviour to students, such a finding is disturbing and has implications for the reputations of the institutions to which the authors are affiliated as well as that of the journals that publish articles that contain plagiarised material.

\section{Introduction}

In 2003, an editorial ${ }^{1}$ in this journal alerted readers to the developing concern about misconduct in the sciences, and acknowledged that the extent of such misconduct and its various manifestations were largely unknown. In 2012. Honig and Bedi ${ }^{2}$ published the findings of a study in the prestigious Academy of Management Learning and Education journal in which they examined 279 papers submitted for the 2009 Academy of Management conference. They found that $25 \%$ of papers contained some degree of plagiarism, with over $13 \%$ evidencing significant plagiarism (defined as comprising $5 \%$ or more of the content). In addition, they reported that a greater amount of plagiarism appeared to emanate from countries outside North America. Against the background of these studies, and given the paucity of research relating to this problem, in the present study, located in a country outside North America, we have attempted to contribute to deliberations in this area.

The objective of the study was to investigate the degree of plagiarism evident in articles published in 2011 in South African management journals that attract subsidy from the Department of Higher Education and Training (DHET). As a subcategory of research dishonesty, plagiarism is the representation of the work of another, or one's own work, without acknowledgement of such work and can include careless paraphrasing, the copying of identical text or providing incomplete references that mislead the reader into believing that the ideas expressed belong to the author of the text. ${ }^{2,3}$

Over the past years student plagiarism has commanded much research attention ${ }^{4-8}$, with increasing focus on the detection of plagiarism ${ }^{9}$ and ways of addressing $i^{4}{ }^{4}$. However, relatively little has been published about plagiarism committed by academics ${ }^{10-13}$, with research thus far regarded as largely anecdotal and speculative ${ }^{2}$. In this regard, Honig and Bedi note:

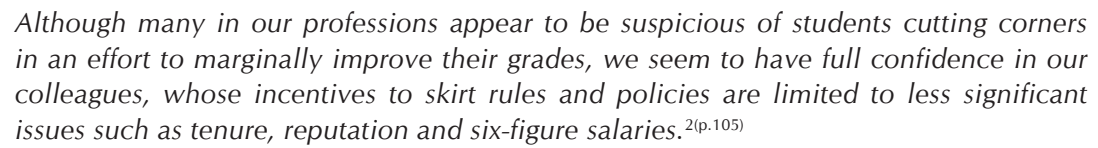

Plagiarism is intellectual theft ${ }^{14}$ and transgresses the fundamental values of the academy ${ }^{15}$, preventing learning, the dissemination of new knowledge, and the integrity of the scientific record ${ }^{16}$. Schminke ${ }^{13}$ notes how plagiarism is sometimes committed by experienced and established authors for whom the blame is apportioned to junior co-authors.

The DHET remits approximately ZAR120 000 to higher education institutions for each peer-reviewed academic article published by a member of the institution in any of the local or international journals that appear on a list compiled by the DHET each year; this funding is an essential income stream for universities. ${ }^{17}$ Accordingly, increasing pressure has been placed on academics to publish in these accredited journals; and such publication is usually linked to financial and promotional rewards. ${ }^{1,18}$ This pressure can contribute to a research culture in which output is promoted at the expense of research quality, which can manifest as plagiarism by those who attempt to achieve the greatest publication output in the shortest time..$^{19}$ In this regard, self-plagiarism - which portrays previous work as new - also contributes to this problem..$^{20}$

Academics have a role to play in developing student moral literacy ${ }^{21}$ and a link has been shown to exist between the dishonesty of academics and student cheating behaviour ${ }^{22}$. Furthermore, academics have been found to be reluctant to report and take action on student academic dishonesty. ${ }^{23}$ Accordingly, it is important to understand research integrity or the lack thereof amongst academics themselves.

\section{Methods}

We submitted 371 peer-reviewed articles that were published in 2011 in 19 South African management journals (spanning the major fields of management) through the Turnitin ${ }^{\mathrm{TM}}$ software program to identify similarities between the articles and other published material, i.e. to identify plagiarism. Once a manuscript is submitted to the program, it is compared against billions of Internet pages, online publications, journal articles and student assignments, dissertations and theses, and a report is generated that highlights the actual text that has been copied and indicates the percentage of similarity between that manuscript and those documents that appear on the Turnitin ${ }^{\mathrm{TM}}$ database. In the remainder of this article, this percentage is referred to as the similarity index. 
Only South African journals that appeared on the Thomson Reuters Web of Science (WoS, previously ISI) or the International Bibliography of the Social Sciences (IBSS) lists or on the local list of journals compiled by the DHET, thereby qualifying for subsidy, were included in the study. Two journals (not included in the 19), containing 17 articles, could not be accessed. The results for each article were checked twice and a conservative approach was adopted in the interpretation of the similarity indices, in which the benefit of doubt was in favour of the authors. For each article, the following content was not included in the assessment of similarity: bibliography/list of references, quotations, strings of words of less than 10, student write-ups on which the article was based, conference proceedings and abstracts detailing the main features of the article. In addition, during the second inspection of the data, specific methodological terms and statistical or mathematical formulae were excluded in the analysis of similarity. The Turnitin ${ }^{T M}$ software program has been used in other studies to detect plagiarism. ${ }^{2,24}$ It has been reported that the Turnitin ${ }^{\mathrm{TM}}$ program itself is conservative in the generation of the results. ${ }^{25}$

\section{Results}

Across the 371 submissions, the similarity index (i.e. the percentage of similarity between an article and the documents in the Turnitin ${ }^{\mathrm{TM}}$ database) ranged from 1 (indicating almost no similarity) to 91 (indicating almost complete similarity). The latter pertained to a single article that was published in two journals under two different titles. Figure 1 shows that the distribution of the similarity index across the 371 submissions was positively skewed. In addition, several outliers were detected, which called for the use of robust statistics in subsequent analyses. ${ }^{26}$ The mean similarity index across the 371 submissions was $17.10(S D=12.15)$, the mode was 9 , the median was 14 and the $20 \%$ trimmed mean was 14.70 (95\% confidence intervals: 13.61 and 15.89 , Winsorised SD=6.67).

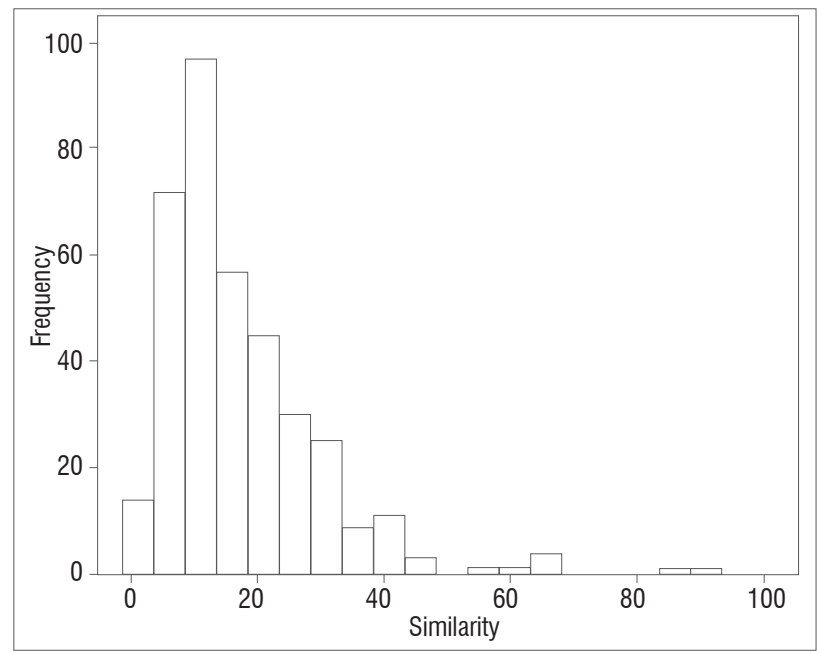

Figure 1: Distribution of the similarity index across 371 submissions.

To gain an overview of the relative frequency of plagiarism we categorised the similarity indices as follows: 1 to 9 as low; 10 to 14 as moderate; 15 to 24 as high and $>24$ as excessive. Table 1 summarises the frequencies in these categories.

Table 1: $\quad$ Similarity according to extent in categories

\begin{tabular}{l|c|c|c}
\hline \hline Category & $\boldsymbol{n}$ & $\%$ & Cumulative $\%$ \\
\hline \hline Low: 1 to 9 & 118 & 31.8 & 31.8 \\
\hline Moderate: 10 to 14 & 73 & 19.7 & 51.5 \\
\hline High: 15 to 24 & 101 & 27.2 & 78.7 \\
\hline Excessive: $25+$ & 79 & 21.3 & 100.0 \\
\hline Total & 371 & 100.0 & \\
\hline
\end{tabular}

The most striking aspect of Table 1 is the proportion of submissions that fell into the high (27.2\% of the submissions) and excessive (21.3\% of the submissions) categories. Whereas one might have expected the bulk of the submissions to fall into the low to moderate categories, the results show that high levels of plagiarism are relatively common in these journals. If we use a cut-off point of $9 \%$ for the similarity index, then it is evident that $68.2 \%$ of the submissions were above the cut-off point. It is noteworthy that $21.3 \%$ of the submissions contained an excessive amount of similarity.

We compared the $20 \%$ trimmed means of the similarity indices among the types of submissions. For submissions to journals in the DHET list $(n=201)$, the trimmed mean $=13.69$ and Winsorised $S D=6.15$; for submissions to journals indexed in WoS $(n=62)$, the trimmed mean $=14.84$ and the Winsorised $S D=5.65$; and for submissions to journals on the IBSS list $(n=108)$, the trimmed mean $=16.71$ and the Winsorised $S D=7.90$. Robust ANOVA $^{26}$ showed that there were no statistically significant differences in the trimmed means across the different journal categories ( $\mathrm{F}=2.2, \mathrm{df} 1=2$, df2 $=96, p=0.11$ ).

We also isolated the 10 journals with at least 20 submissions during the period under review ( $n=270$ submissions). Across these journals the trimmed means of the similarity index ranged from 11.67 to 27.24 . Robust ANOVA ${ }^{26}$ revealed statistically significant differences in the trimmed means ( $\mathrm{F}=2.6, \mathrm{df} 1=9$, df2 $=62, p=0.012)$, with a medium effect size $(\xi=0.40)$. Robust post-hoc tests ${ }^{26}$ revealed that the differences could be traced to excessively high levels of similarity in one journal only (i.e. the journal with a trimmed mean similarity index of 27).

We also examined whether single versus multiple authorship played a role in the similarity index of an article. The difference in trimmed means between three categories of authorship - single $(n=169$, trimmed mean $=15.75$, Winsorised $S D=6.76)$, dual $(n=148$, trimmed mean $=15.42$, Winsorised $S D=7.08)$ and three or more authors $(n=54$, trimmed mean $=10.65$, Winsorised $S D=4.28$ ) - was statistically significant $(\mathrm{F}=9.6 \mathrm{df} 1=2, \mathrm{df} 2=115, p=0.0001)$ with a medium effect size $(\xi=0.32)$. Robust post-hoc tests revealed that the similarity index of articles with three or more authors was significantly smaller than that of a single or dual authored article. No significant difference between single and dual authored articles was observed.

We complemented the three robust analyses of variance reported above with standard analyses of variance and non-parametric KruskalWallis tests, both of which yielded a similar pattern of results as the robust tests.

\section{Discussion}

Our results indicate that there was extensive plagiarism in 19 South African management journals during the period under review, confirming the findings of other studies.,10-13 The findings also indicate that although one journal appeared to contain more plagiarised articles than the others, the problem of plagiarism existed across the board. The type of journal (i.e. whether it appears on the DHET, WoS or IBSS lists) was not a factor in the level of plagiarism. However, the findings indicated that articles submitted by three or more authors contained significantly less plagiarised material than did those articles submitted by a single or by dual authors. A possible explanation for this finding is that potential plagiarism can be more readily detected and corrected when several authors are involved. Conversely, a single author may more easily be able to hide plagiarised work.

We suggest that the intense pressure on universities and their academics to increase their research output within short time periods, plays a role in this problem. In addition, academics are rewarded in a variety of ways for such output ${ }^{1,19}$, which can contribute to a culture of expedience and opportunism ${ }^{18}$.

An additional problem of governance also emerges when one considers the payment of government subsidy to universities based on research output. If at least one author of an article is affiliated to a South African higher education institution, government will pay a research subsidy of ZAR120 000 per article, which may be proportionally split according to 
the institutional affiliation of authors. Excluding those articles submitted by authors not affiliated to a South African higher education institution $(n=47)$, it was estimated that government paid ZAR32 400000 in subsidies for articles published in these 19 journals during the period under review. Given that $21.3 \%$ of these articles contained excessive plagiarism, a government subsidy of almost ZAR7 000000 was paid for questionable publications.

The problem of human error in data coding always exists in studies such as this one, but we tried to minimise this risk by checking the data twice. The findings indicate the existence of plagiarism in the published articles we submitted for study. This finding has implications for government, for the universities to which the authors are affiliated and for the journals themselves.

The culture of research expediency that may be developing in academic institutions in order to increase subsidised research output can have longterm implications for the reputation of universities. Their contribution to society can also be compromised in terms of both the dissemination of new knowledge and the upholding of moral values transmitted through the students who graduate from these institutions and who can be expected to be influenced by unethical role models. ${ }^{22}$ It is critical that the DHET engages with universities to devise measures to subsidise research output without inadvertently promoting the sacrificing of the quality of such research and inadvertently encouraging shortcuts, such as plagiarism. In a similar vein, internal rewards to academics should not be based on the quantity of research output without considering that a greater contribution could be made by researchers who publish fewer articles but in highly cited journals with greater stringency in requirements pertaining to quality. It is also recommended that, in order to preserve the reputation of journals, editors subject manuscripts to plagiarism detection through software programs and that the penalties for detected plagiarism be severe for authors.

It is recommended that future studies of this nature explore the extent of plagiarism (if any) in journals related to other disciplines in order to ascertain whether this problem is pervasive in other fields as well. In addition, a qualitative study of the experiences of journal editors in addressing plagiarism may throw some light on how the extent of plagiarism, noted in this study, managed to appear in articles that are deemed to contain original material for which the DHET remits subsidy to academic institutions.

\section{Acknowledgements}

We thank Ms Ziyanda Makupula for her assistance in sourcing some of the literature used in the article and Mr Richard Devey of Statkon at the University of Johannesburg for assistance with the initial data analysis.

\section{Authors' contributions}

A.T. was the project leader and was responsible for the literature review and the data collection. G.P.d.B. conducted the data analysis. Both authors were involved in the interpretation of the data and the writing of the manuscript.

\section{References}

1. The good, the bad, and the ugly. S Afr J Sci. 2003;99(9-10):402-403.

2. Honig B, Bedi A. The fox in the hen house: A critical examination of plagiarism among members of the Academy of Management. Acad Manag Learn Educ. 2012;11(1):101-123. http://dx.doi.org/10.5465/amle.2010.0084

3. Bedeian AG, Taylor SG, Miller AN. Management science on the credibility bubble: Cardinal sins and various misdemeanours. Acad Manag Learn Educ. 2010;9(4):715-725. http://dx.doi.org/10.5465/AMLE.2010.56659889

4. Kisamore JL, Stone TH, Jawajar IM. Academic integrity: The relationship between individual and situational factors on misconduct contemplations. J Bus Ethics. 2007;75(4):381-394. http://dx.doi.org/10.1007/s10551-0069260-9
5. Levy ES, Rakovski CC. A zero tolerance professor and student registration choices. Res High Educ. 2006;47(6):735-754. http://dx.doi.org/10.1007/ s11162-006-9013-8

6. McCabe DL, Treviňo LK, Butterfield KD. Honor codes and other contextual influences on academic integrity. Res High Educ. 2002;43(3):357-378. http://dx.doi.org/10.1023/A:1014893102151

7. Rawwas M, Swaidan Z, Isakson H. A comparative study of ethical beliefs of Master of Business Administration students in the United States with those in Hong Kong. J Educ Bus. 2007;82(3):146-158. http://dx.doi.org/10.3200/ JOEB.82.3.146-158

8. Wasley P. The plagiarism hunter. Chron High Educ. 2006;52(49):A8-A11.

9. Drinan P, Bertram Gallant T. Plagiarism and academic integrity systems. $J$ Libr Admin. 2008;47(3-4):125-140. http://dx.doi.org/10.1080/ 01930820802186472

10. Clarke R. Plagiarism by academics: More complex than it seems. J Assoc Inform Syst. 2006;7(2):91-121.

11. Shahabuddin S. Plagiarism in academia. Int J Teach Learn High Educ. 2009;21(3):353-359

12. Kacmar KM. From the editors: An ethical quiz. Acad Manag J. 2009;52(3):432434. http://dx.doi.org/10.5465/AMJ.2009.41330319

13. Schminke S. Editor's comments: The better angle of our nature - ethics and integrity in the publishing process. Acad Manag Rev. 2009;34(4):586-591. http://dx.doi.org/10.5465/AMR.2009.44882922

14. Hansen B, Stith S, Tesdell LS. Plagiarism: What's the big deal? Bus Comm Q. 2011;74(2):188-191. http://dx.doi.org/10.1177/1080569911404695

15. Lewis BR, Duchac JE, Beets SD. An academic publisher's response to plagiarism. J Bus Ethics. 2011;102:489-506. http://dx.doi.org/10.1007/ s10551-011-0827-8

16. Yentis SM. Editorial: Another kind of ethics: From corrections to retractions. Anaesthesia. 2010;65(12):1163-1172. http://dx.doi.org/10.1111/j.13652044.2010.06557.x

17. Muller S. Publish or perish - and damage too? Mail and Guardian Education Supplement. 2012 April 13-19;40.

18. Atkins J, Herfel W. Counting the beans in the degree factory. Int J Educ Integ. 2006;2(1):3-12.

19. Kelley PC, Chang PL. A typology of university ethical lapses: Types, levels of seriousness, and originating location. J High Educ. 2007;78,4:402-429. http://dx.doi.org/10.1353/jhe.2007.0024

20. Bretag T, Mahmud S. Self-plagiarism or appropriate textual re-use? J Acad Ethics. 2009;7:193-205. http://dx.doi.org/10.1007/s10805-009-9092-1

21. Osiemo LB. Developing responsible leaders: The university at the service of the persons. J Bus Ethics. 2012;108:131-143. http://dx.doi.org/10.1007/ s10551-011-1087-3

22. Schnake M, Dumler MP, Fredenberger W. Predicting overall ethical climate, student retention, cheating, satisfaction with university, and perceived stress with student perceptions of faculty unethical behavior. Acad Educ Leader J. 2005;9(3):31-39.

23. Thomas A, De Bruin GP. Student academic dishonesty: What do academics think and do and what are the barriers to action? Afr J Bus Ethics. 2012;6(1):13-24. http://dx.doi.org/10.4103/1817-7417.104698

24. Stapleton P. Gauging the effectiveness of anti-plagiarism software: An empirical study of second language graduate writers. J Eng Acad Pur. 2012;11: 125-133. http://dx.doi.org/10.1016/j.jeap.2011.10.003

25. Fiedler RL, Kaner C. Plagiarism detection services: How well do they actually perform? IEEE Tech Soc Mag. 2010;29(4):37-43. http://dx.doi.org/10.1109/ MTS.2010.939225

26. Wilcox R. Modern statistics for the social and behavioral sciences: A practical introduction. Boca Raton, FL: CRC Press; 2012. 